\title{
Ultrasound Guided Platelet Rich Plasma and Autologous Stem Cells Injections: An Emerging Alternative Treatment for Osteochondral Defects
} Susmarski AJ1, Franco $\mathrm{T}^{2}$ and De Luigi $\mathrm{AJ}^{3 *}$

${ }^{1}$ United States Navy, USA

${ }^{2}$ Medical Rehabilitation Inc., USA

${ }^{3}$ MedStar National Rehabilitation Hospital MedStar Georgetown University Hospital, USA

\begin{abstract}
Osteochondral defects are a frequent cause of knee pain in the population. The majority of the diagnosed cases of osteochondral defects (OCD) are treated with surgical procedures. However, there are emerging alternative and integrative medicine options which have appeared on the horizon. These alternative treatment options are minimally invasive with a significant cost reduction and the potential for an earlier return on function due to a shorter rehabilitation period. The focus of this report will be to demonstrate the efficacy of Ultrasound (US) guided Platelet Rich Plasma (PRP) and Autologous Stem Cell Injections in the treatment of osteochondral Defects.
\end{abstract}

Keywords: Platelet rich plasma; Stem cells; Osteochondral defect

\section{Introduction}

An osteochondral defect is an area inside a joint where a portion of the joint cartilage and underlying bone has been lost [1]. This poses a significant and increasing health care concern due to hyaline cartilage tissues inability to spontaneously regenerate in vivo [2]. The current most common surgical procedure is an arthroscopic knee microfracture technique popularized by Steadman et al. [3] which relies on the release of autologous stem cells at the site of the defect to initiate fibrocartilage repair [4]. The current most progressive strategy to repair the articular cartilage defect is to fill an osteochondral defect with a tissue-engineered cartilage-like tissue or a cell-seeded scaffold material [5].

Autologous Stem Cell Transplantation is a process in which stem cells from the bone marrow or blood are withdrawn and the removed cells are reinfused to form a new population of blood cells [6]. Mesenchymal stem cells (MSC) are pluripotent cells found in multiple human adult tissues including bone marrow, synovial tissues, and adipose tissues [7]. MSCs are derived from the mesoderm and have been shown to differentiate into bone, cartilage, muscle, and adipose tissue [7].

Platelets are known to contain key growth factors such as Platelet Derived Growth Factors (PDGFs), Transforming Growth Factors (TGFs), Fibroblast Growth Factors (FGFs), and Interleukins (IL) [8]. Commercially available centrifugation systems can isolate and concentrate platelets from a venous blood sample and the resulting blood isolate is known as Platelet Rich Plasma (PRP) [4]. Most commercially available PRP centrifuge products have the ability to produce a 4-7 fold increase in platelet concentration compared to whole blood [4]. During injection needle "stimulus" (fenestration) is used to induce and stimulate a biologic inflammatory response, promote repair, and further recruit/activate circulation derived cells [9]. In vitro studies have demonstrated PRP's ability to inhibit excess inflammation, as well as, augment mesenchymal stem cell proliferation and maturation [10]. PRP has also been found to induce type 1 collagen [10], influence chondrogenic differentiation [11], and contribute to hyaluronic acid secretion after intra-articular injections [12]. Furthermore, skeletal muscle derived stem cells-in vitro-were shown to have enhanced osteogenic activity and differentiation in conjunction with PRP [13].

There is a wide variety in the literature in regards to interval between injections, however, the current recommendation proposed by Nguyen et al. [14] is an approximately six to eight week interval between injections and perhaps even longer in regard to chronic conditions. Wound healing is in an active state for a minimum of six weeks and remodeling has potential to occur over a time frame of months.

We present a patient who was initially diagnosed with a meniscal injury, as well as, a prominent osteochondral defect. The patient underwent surgery for the meniscal injury; however, had persistent refractory knee pain secondary to the osteochondral defect. Prior to undergoing another surgery, the patient sought consultation regarding less invasive treatment options including PRP and Autologous Stem Cell injections.

\section{Case Report}

A 58 year old male athlete who sustained a meniscal tear and upon arthroscopy for partial meniscectomy, as well as, Magnetic Resonance Imaging (MRI) was found to have a significant osteochondral defect of his left medial femoral condyle (Figure 1). Since his injury, the patient had been sidelined and unable to resume play on a competitive baseball team. He presented to the Sports Medicine clinic with persistent pain of two and one-half years since his initial injury to discuss additional treatment options including Platelet Rich Plasma (PRP) injections and Autologous Stem Cell injection before proceeding with a proposed osteotomy with varus deformity correction [15].

Physical Examination of his left knee revealed moderate to severe tenderness over the left medial femoral condyle with the knee fully flexed. The patient possessed full active range of motion with left knee flexion and extension. There was bilateral lower extremity varus deformity (left greater than right). There was not any effusion noted

*Corresponding author: Arthur Jason De Luigi, MedStar National Rehabilitation Hospital MedStar Georgetown University Hospital, USA, E-mail: ajweege@yahoo.com

Received January 24, 2013; Accepted March 11, 2013; Published March 14, 2013

Citation: Susmarski AJ, Franco T, De Luigi AJ (2013) Ultrasound Guided Platelet Rich Plasma and Autologous Stem Cells Injections: An Emerging Alternative Treatment for Osteochondral Defects. Altern Integ Med 2: 108. doi:10.4172 2327-5162.1000108

Copyright: (c) 2013 Susmarski AJ, et al. This is an open-access article distributed under the terms of the Creative Commons Attribution License, which permits unrestricted use, distribution, and reproduction in any medium, provided the original author and source are credited. 


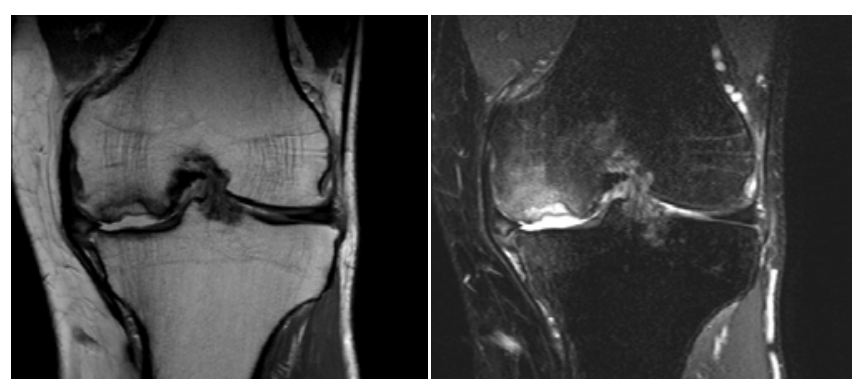

"Prominent" osteochondral defect on weight bearing surface of medial femora condyle.

Figure 1: MRI Left Knee (April 11, 2008)

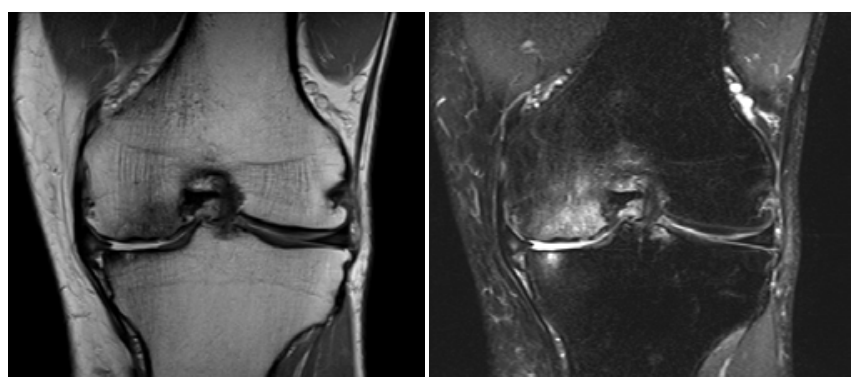

The previously seen osteochondral defect is now "barely evident"

Figure 2: MRI Left Knee (August 17, 2010).

in the left knee. Special tests of the knee did not produce symptoms with McMurray's test, and there was a firm end point with Lachman's, anterior and posterior drawer testing, as well as, valgus and varus testing.

The patient was diagnosed with a prominent left medial femoral condyle osteochondral defect. The patient was seeking non-surgical treatment options and consented to an ultrasound (US) guided PRP injection. On follow-up evaluation the patient had partial improvement of symptoms, however, was still unable to resume full activities and return to play. Nearly five months after the initial PRP injection, the patient underwent an US guided Autologous Stem Cell injection followed by another US guided PRP injection approximately two months later (seven months from the initial PRP injection). Injections were performed by first identifying the osteochondral defect under ultrasound. Once the lesion was localized under ultrasound, a 25 gauge, 1.5 inch needle was used to trephinate the defect on the distal femur. Injections with PRP and Autologous Stem Cells were then made while maintaining visualization of the defect by ultrasound. Finally, hemostasis was obtained, a band-aid and compressive dressing placed, and the patient was able to ambulate out of the exam room without difficulty.

Two months following the last injection and 28 months from the original MRI, repeated imaging of the knee revealed a significant interval change from a "prominent" osteochondral defect to one that was "barely evident" (Figure 2). Additionally, the patient reported marked improvement in pain and discomfort allowing him to resume full activities (competitive baseball) without restriction one month later and without any re-exacerbation of symptoms.

\section{Discussion}

This case study presents a clinical scenario in which an athlete was able to achieve resolution of his symptoms and return to play through a combination of US guided PRP and Autologous Stem Cell injections.

In regards to osteochondral defects, there were currently no randomized control trials utilizing PRP at the time of submission of this article. However, there were a handful regarding osteoarthritis in humans and intriguing studies regarding animal subjects [14].

There has been a study by Qi et al. [16], through the utilization of rabbit models with full-thickness cartilage defects in the patellar groove, were able to create the induction of cartilage tissues with the use of PRP in conjunction with a collagen matrix (control group was collagen matrix without PRP).

In another study by Sun et al. [15], rabbit models with articular cartilage defects of the femoro-patellar groove were treated with PRP and a polyacticglycolic acid (PLGA) scaffolding versus PLGA alone versus no treatment. Their studies showed that the PRP with PLGA scaffold stimulated osteochondral formation and Type II collagen accumulation on histologic and micro-CT evaluation. Furthermore, they also were able to demonstrate underlying trabecular bony growth.

Studies on the effect of Autologous Stem Cells have also been conducted in rabbit models. Wakitani et al [17]. in 1994 performed a study where Autologous Stem Cells were isolated, grown in vitro, and dispersed in a collagen type 1 gel which initiated the repair of the articular cartilage and the reformation of subchondral bone (using contra-lateral knee defects as a control).

There is a single human case study conducted by Centeno et al. [4] of a 46 year old male with MRI proven Degenerative Joint Disease (DJD) underwent who bone marrow aspiration of the iliac crest. MSCs were later injected into the subject's knee resulting in statistically significant meniscus and cartilage growth on MRI and subjective improvement in symptoms 24 weeks post injection.

The potential benefits of cell placement by US guided injection versus surgery should also be taken into consideration when determining the appropriate treatment regimen for a patient with an osteochondral defect. US guidance allows for the cells to be placed directly at the area of the visualized deficit without creating any further damage (microfracture) at the injured site [18] There is a reduced risk of infection and complications as the procedure does not require general anesthesia and is inherently less invasive in nature when compared to Orthopaedic microfracture surgery. Furthermore, the decrease in procedure and subsequent recovery time increases convenience for physician and patient, reduces time off from training, as well as, allows for quicker return to athletics.

There is also significant financial benefit of US guided injections versus orthopedic microfracture surgery for osteochondral defect treatment. US guided PRP injections are currently not covered by most healthcare insurers, but this minimally invasive in office procedure cost substantially less than operative management. Filardo et al. [9] found the surgical procedure of microfracture to be more expensive than autologous chondrocyte implantation. Given the current healthcare climate in the United States healthcare system US guided treatment regimens for the treatment of osteochondral defects in patients will lead to a significant reduction of cost.

Potential drawbacks of treatment with PRP injections can lie within median duration of treatment, i.e. clinical improvement of 
Citation: Susmarski AJ, Franco T, De Luigi AJ (2013) Ultrasound Guided Platelet Rich Plasma and Autologous Stem Cells Injections: An Emerging Alternative Treatment for Osteochondral Defects. Altern Integ Med 2: 108. doi: 10.4172/2327-5162.1000108

symptoms, in addition to the potential for inability to provide definitive care for osteochondral defects. Filardo et al. [9] found that during their 90 plus intraarticular PRP knee injections the median duration of clinical improvement was 9 months. Furthermore, cessation of clinical improvement after 9 months may result in need for reinjection (exposure to another interventional procedure and associated cost) and/or the patient may ultimately need to have a more invasive surgical intervention in order to attempt to achieve definitive resolution of symptoms. Duration of therapeutic effect and degree of clinical benefit has also been correlated with longer lasting results in younger, male, and lower degree of cartilage degeneration versus decreased efficacy identified in female patients and those with a higher BMI [10].

While the autologous osteochondral transplantation has been shown to have the ability/potential to improve cartilaginous defects the invasiveness of the procedure and procurement of the Autologous Stem Cells has been identified as a potential concern and limitation for repair of larger defects [19].

Most human studies to date are either case series or retrospective studies, as well as, often small in size in regards to number of patients and subsequently have an associated lack of power. A clear and standardized treatment protocol for PRP or Autologous Stem Cell injections has not been clearly established/agreed upon to date due to the aforementioned limited data; however, as mentioned above, Nguyen et al. in their comprehensive literature review have begun to identify and propose potential standardization of potential treatment protocols [14].

\section{Conclusion}

Overall, the patient had both subjective symptomatic improvements of symptoms, as well as, significant radiographic evidence of improvement of the osteochondral defect resulting in return to competitive sport. At this time, PRP injections have been showed to advance the healing process in various musculoskeletal ailments, and it appears that supplementation with Autologous Stem Cells may be a promising treatment option in the treatment of osteochondral defects.

Future considerations of prospective randomized trials utilizing PRP, Autologous Stem Cell injections, as well as the combination of the these treatments should be performed to establish definitive results for the treatment of osteochondral defects in humans.

\section{References}

1. Osteochondral Defect retrieved 16 February 2011.

2. Mandelbaum BR, Browne JE, Fu F, Micheli L, Mosely JB Jr, et al. (1998) Articular cartilage lesions of the knee. Am J Sports Med 26: 853-861.

3. Steadman JR, Ramappa AJ, Maxwell RB, Briggs KK (2007) An arthroscopic treatment regimen for osteoarthritis of the knee. Arthroscopy 23: 948-955.

4. Centeno CJ, Busse D, Kisiday J, Keohan C, Freeman M, et al. (2008) Increased knee cartilage volume in degenerative joint disease using percutaneously implanted, autologous mesenchymal stem cells. Pain Physician 11: 343-353.

5. Brittberg M, Lindahl A, Nilsson A, Ohlsson C, Isaksson O, et al. (1994) Treatment of deep cartilage defects in the knee with autologous chondrocyte transplantation. N Engl J Med 331: 889-895.

6. Autologous stem cell transplantation (2009) Mosby's Medical Dictionary (8thedn).
7. Szilvassy SJ (2003) The biology of hematopoietic stem cells. Arch Med Res 34: 446-460.

8. Fréchette JP, Martineau I, Gagnon G (2005) Platelet-rich plasmas: growth factor content and roles in wound healing. J Dent Res 84: 434-439.

9. Filardo G, Kon E, Della Villa S, Vincentelli F, Fornasari PM, et al. (2010) Use of platelet-rich plasma for the treatment of refractory jumper's knee. Int Orthop 34: 909-915.

10. Kajikawa Y, Morihara T, Sakamoto H, Matsuda K, Oshima Y, et al. (2008) Platelet-rich plasma enhances the initial mobilization of circulation-derived cells for tendon healing. J Cell Physiol 215: 837-845.

11. Mishra A, Tummala P, King A, Lee B, Kraus M, et al. (2009) Buffered plateletrich plasma enhances mesenchymal stem cell proliferation and chondrogenic differentiation. Tissue Eng Part C Methods 15: 431-435.

12. Anitua E, Sanchez M, Nurden AT, Zalduendo MM, de la Fuente M, et al. (2007) Platelet-released growth factors enhance the secretion of hyaluronic acid and induce hepatocyte growth factor production by synovial fibroblasts from arthritic patients. Rheumatology (Oxford) 46: 1769-1772.

13. Shan L, Wang G, Zhang C, Zeng B (2009) Effect of various concentrations of platelet-rich plasma on osteogenic differentiation of skeletal muscle-derived stem cells. Zhongguo Xiu Fu Chong Jian Wai Ke Za Zhi 23: 991-996.

14. Nguyen RT, Borg-Stein J, McInnis K (2011) Applications of platelet-rich plasma in musculoskeletal and sports medicine: an evidence-based approach. PM R 3: $226-250$.

15. Sun Y, Feng Y, Zhang CQ, Chen SB, Cheng XG (2010) The regenerative effect of platelet-rich plasma on healing in large osteochondral defects. Int Orthop 34: 589-597.

16. Qi YY, Chen X, Jiang YZ, Cai HX, Wang LL, et al. (2009) Local delivery of autologous platelet in collagen matrix simulated in situ articular cartilage repair. Cell Transplant 18: 1161-1169.

17. Wakitani S, Goto T, Pineda SJ, Young RG, Mansour JM, et al. (1994) Mesenchymal cell-based repair of large, full-thickness defects of articular cartilage. J Bone Joint Surg Am 76: 579-592.

18. Deangelis JP, Spindler KP (2010) Traumatic Bone Bruises in the Athlete's Knee. Sports Health 2: 398-402.

19. Koga H, Shimaya M, Muneta T, Nimura A, Morito T, et al. (2008) Local adheren technique for transplanting mesenchymal stem cells as a potential treatment of cartilage defect. Arthritis Res Ther 10: R84. 